\title{
On the Group Controllability of Leader-Based Continuous-Time Multiagent Systems
}

\author{
Bo Liu $\left(\mathbb{D},{ }^{1}\right.$ Licheng Wu, ${ }^{1}$ Rong Li, ${ }^{2}$ Housheng Su $\mathbb{D},{ }^{3}$ and Yue Han ${ }^{4}$ \\ ${ }^{1}$ School of Information Engineering, Minzu University of China, Beijing 100081, China \\ ${ }^{2}$ School of Statistics and Mathematics, Shanghai Lixin University of Accounting and Finance, Shanghai 201209, China \\ ${ }^{3}$ Key Laboratory of Imaging Processing and Intelligence Control, School of Artificial Intelligence and Automation, \\ Huazhong University of Science and Technology, Wuhan 430074, China \\ ${ }^{4}$ College of Science, North China University of Technology, Beijing 100144, China
}

Correspondence should be addressed to Bo Liu; boliu@ncut.edu.cn and Housheng Su; houshengsu@qq.com

Received 11 January 2020; Accepted 7 April 2020; Published 15 May 2020

Academic Editor: Cornelio Posadas-Castillo

Copyright (C) 2020 Bo Liu et al. This is an open access article distributed under the Creative Commons Attribution License, which permits unrestricted use, distribution, and reproduction in any medium, provided the original work is properly cited.

The group controllability of continuous-time multiagent systems (MASs) with multiple leaders is considered in this paper, where the entire group is compartmentalized into a few subgroups. The group controllability concept of continuous-time MASs with multiple leaders is put forward, and the group controllability criteria are obtained for switching and fixed topologies, respectively. Finally, the numerical simulations are given to prove the validity of the theoretical results.

\section{Introduction}

In recent decades, distributed coordination control of networked MASs has become a hot and challenging issue in lots of areas, such as applied mathematics, control theory, mechanics, engineering, and neurobiology [1-10]. Studies in this area include several basic problems, such as stability, consensus and synchronization [11], containment [12], controllability [13], and formation control and tracking control [14].

The controllability problem is a key essential problem in modern control theory and attracts increasing attention due to its wide applications in engineering. An MAS is controllable if each dynamic follower can attain its desirable configuration from any initial state during finite time by regulating some leaders. In [15], Tanner first put forward the controllability problem of networked systems in a leader-following framework, where a certain agent was acted as the leader (the external control input), and an algebraic feature based on eigenvalues and eigenvectors of such system's Laplacian matrices was derived by nearest neighbor rules. Based on this, Liu et al. [16] discussed the controllability of discrete-time MASs with a single leader based on nearest neighbor rules and derived a simple controllable condition for such a system on switching topology. Afterwards, further studies on the controllability of MASs have mainly been concentrated from graph-theoretic and algebraic-theoretic points of view, respectively. At present, many works on the controllability of MASs from the perspective of graph theory have concentrated on the basis of partitions of graph topology, such as equitable partition/relaxed equitable partition/ external equitable partition in [17], connected component partition in [18], and selection of leaders [19]. Further research studies on the controllability were presented for some different special topology graphs, such as path graphs [20], cycle graphs [21], multichain topologies [22], stars and trees [23], two-time-scale topologies [24], and regular graphs [25]. Lots of algebraic controllable conditions of MASs were characterized in $[26,27]$.

The aforementioned results on the controllability of MASs just contained a single group. However, in engineering practice, a single group can be compartmentalized into some subgroups with the improvement of MASs' 
complexity [28]. It is a very challenging work to study the controllability problem of MASs with multiple subgroups and multiple leaders considering the control law, the information topology structure between different subgroups, and the effect of dynamical leaders acting on the follower agents, which will be highlighted in this paper. More recently, the group controllability of continuous-time/discrete-time MASs leaderless with different topologies and communication restrictions in $[29,30]$ was studied, respectively.

Motivated by the results of previous studies, this paper aims at the group controllability of continuous-time MASs consisting of some different subgroups by adjusting the leaders. The main contributions of this paper are summed up as follows:

(1) Different from the group controllability problem of continuous-time MASs under the leaderless framework studied based on the fixed topology in [30], the current work has considered the group controllability of continuous-time MASs under the leaderfollower framework with fixed topology and switching topology, respectively, which can be expressed by the system matrices. It is obvious that different models can lead to completely different features for MASs with leaders.

(2) The concepts of the group controllability of continuous-time MASs with multiple leaders are proposed based on switching and fixed topologies, respectively.

(3) Sufficient and/or necessary algebraic- and graphtheoretic group controllable characterizations of continuous-time MASs with multiple leaders under the group consensus protocol are established from the system's Laplacian matrices.

(4) The effects of subgroups and leaders on the group controllability are discussed.

The rest of this work is arranged as follows. The problem formulation is stated in Section 2. Section 3 builds the group controllability of MASs with multiple leaders. Numerical example and simulations are given in Section 4. Finally, Section 5 summarises the conclusion.

\section{Problem Formulation}

Consider a continuous-time MAS consisting of $N$ agents governed by

$$
\dot{x}_{i}(t)=u_{i}(t), \quad i=1, \ldots, N,
$$

where $x_{i} \in R$ is the state and $u_{i} \in R$ is the control input, respectively.

In engineering practice, the whole group can be compartmentalized into a few subgroups. Without loss of generality, in this paper, such MAS consisting of $m+n+$ $l+k(m, n, l, k>1)$ agents is compartmentalized into subgroup $\left(\mathscr{G}_{1}, x^{1}\right)$ and subgroup $\left(\mathscr{G}_{2}, x^{2}\right)$, as shown in Figure 1.

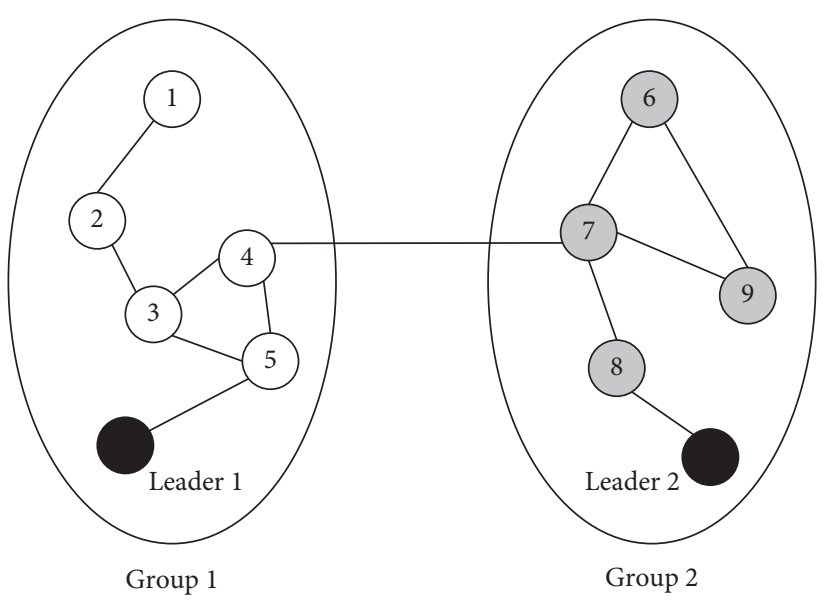

Figure 1: Topology $\mathscr{G}$.

Denote $\ell_{1} \triangleq\{1, \ldots, m\}, \ell_{2} \triangleq\{m+1, \ldots, m+n\}, \ell_{1_{l}} \triangleq\{m+$ $n+1, \ldots, m+n+l\}, \quad \ell_{2_{l}} \triangleq\{m+n+l+1, \ldots, m+n+l+k\}$, $\mathscr{V}_{1} \triangleq\left\{v_{1}, \ldots, v_{m}\right\}$, and $\mathscr{V}_{2} \triangleq\left\{v_{m+1}, \ldots, v_{m+n}\right\}$; then, $\ell=\ell_{1} \cup \ell_{2}, \ell_{l}=\ell_{1_{l}} \cup \ell_{2_{l}}$, and $\mathscr{V}=\mathscr{V}_{1} \cup \mathscr{V}_{2} . \mathscr{N}_{i}$ is the $i$ th agent's neighbor set with $\mathscr{N}_{1 i}=\left\{v_{j} \in \mathscr{V}_{1}:\left(v_{j}, v_{i}\right) \in \mathscr{E}\right\}$ and $\mathcal{N}_{2 i}=\left\{v_{j} \in \mathscr{V}_{2}:\left(v_{j}, v_{i}\right) \in \mathscr{E}\right\}$, where $\mathcal{N}_{i}=\mathscr{N}_{1 i} \cup \mathscr{N}_{2 i}$ and $\mathcal{N}_{1 i} \cap \mathscr{N}_{2 i}=\varnothing . \mathscr{N}_{1 p}$ and $\mathcal{N}_{2 p}$, respectively, represent the leaders' neighbor sets of subgroups 1 and 2 .

Inspired by [30], the control input $u_{i}$ is designed as follows:

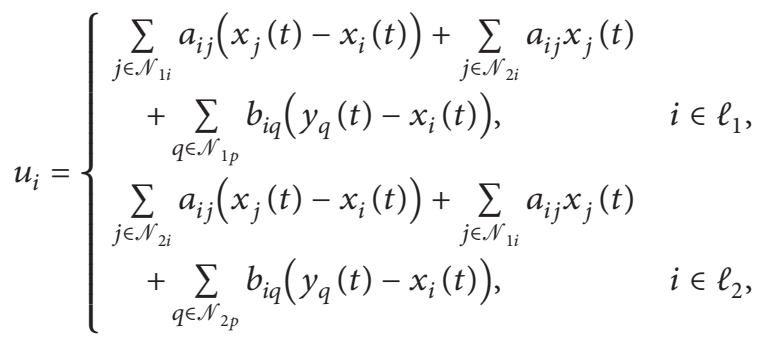

where $a_{i j} \in R, b_{i q} \geq 0, \forall i, j, q \in \ell_{1}, \ell_{2}$.

Remark 1. It is noted that the $(i, j)$ th entry of the system's adjacency matrix, denoted as $a_{i j}$, in this paper, can be allowed to be negative, which makes it more difficult and complex to discuss the group controllability problem since there are negative factors in the coupling links between different subgroups.

Supposed that $x^{1} \triangleq\left(x_{1}, \ldots, x_{m}\right)^{T}$ and $x^{2} \triangleq\left(x_{m+1}, \ldots, x_{m+n}\right)^{T}$ are the state vectors of follower agents in $\mathscr{G}_{1}$ and $\mathscr{G}_{2}$ and $y^{1} \triangleq\left(y_{1}, \ldots, y_{l}\right)^{T}$ and $y^{2} \triangleq\left(y_{l+1}, \ldots, y_{l+p}\right)^{T}$ are the state vectors of the leader agents in $\mathscr{G}_{1}$ and $\mathscr{G}_{2}$, respectively. Then, the dynamics of the followers in system (1) becomes

$$
\begin{aligned}
{\left[\begin{array}{c}
\dot{x}^{1} \\
\dot{x}^{2}
\end{array}\right] } & =\left[\begin{array}{cc}
-L_{1}-R_{1} & C_{1} \\
C_{2} & -L_{2}-R_{2}
\end{array}\right]\left[\begin{array}{l}
x^{1} \\
x^{2}
\end{array}\right]+\left[\begin{array}{cc}
B_{1} & 0 \\
0 & B_{2}
\end{array}\right]\left[\begin{array}{l}
y^{1} \\
y^{2}
\end{array}\right] \\
& \triangleq\left[\begin{array}{ll}
A_{1} & C_{1} \\
C_{2} & A_{2}
\end{array}\right]\left[\begin{array}{l}
x^{1} \\
x^{2}
\end{array}\right]+\left[\begin{array}{cc}
B_{1} & 0 \\
0 & B_{2}
\end{array}\right]\left[\begin{array}{l}
y^{1} \\
y^{2}
\end{array}\right]
\end{aligned}
$$


where $L_{1}=\left[l_{i j}\right] \in \mathbb{R}^{m \times m}$ and $L_{2}=\left[l_{i j}\right] \in \mathbb{R}^{n \times n}$ are the Laplacian matrices of graphs $\mathscr{G}_{1}$ and $\mathscr{G}_{2}$, respectively, $R_{1}=\operatorname{diag}\left(\sum_{q \in \mathcal{N}_{1 q}} b_{1 q}, \ldots, \sum_{q \in \mathcal{N}_{1 q}} b_{m q}\right) \in \mathbb{R}^{m \times m}, \quad R_{2}=\operatorname{diag}$ $\left(\sum_{q \in \mathcal{N}_{2 q}} b_{m+1, q}, \ldots, \sum_{q \in \mathcal{N}_{2 q}} b_{m+n, q}\right) \in \mathbb{R}^{n \times n}$,

$$
\begin{aligned}
& A_{1} \triangleq-L_{1}-R_{1} \in \mathbb{R}^{m \times m}, \quad B_{1} \in \mathbb{R}^{m \times l}, C_{1} \in \mathbb{R}^{m \times n}, \\
& A_{2} \triangleq-L_{2}-R_{2} \in \mathbb{R}^{n \times n}, \quad B_{2} \in \mathbb{R}^{n \times k}, C_{2} \in \mathbb{R}^{n \times m} .
\end{aligned}
$$

Remark 2. Furthermore, because $a_{i j}$ can be allowed to be negative, nonzero controller gains can be appropriately selected as long as $L_{1}$ and $L_{2}$ are Laplacian matrices. In essence, the group controllability of continuous-time leaderbased MASs cannot be affected by the controller gains.

In order to discuss the group controllability problem of system (3), its equivalent augmented system can be described by

$$
\left\{\begin{array}{l}
\dot{x}^{1}=A_{1} x^{1}+\left[\begin{array}{ll}
C_{1} & B_{1}
\end{array}\right]\left[\begin{array}{l}
x^{2} \\
y^{1}
\end{array}\right], \\
\dot{x}^{2}=A_{2} x^{2}+\left[\begin{array}{ll}
C_{2} & B_{2}
\end{array}\right]\left[\begin{array}{l}
x^{1} \\
y^{2}
\end{array}\right],
\end{array}\right.
$$

where $\left(x^{2}, y^{1}\right)$ and $\left(x^{1}, y^{2}\right)$ are the inputs of subgroup $\left(\mathscr{G}_{1}, x^{1}\right)$ and subgroup $\left(\mathscr{G}_{2}, x^{2}\right)$, respectively.

\section{Group Controllability Analysis}

This section discusses the group controllability of continuous-time leader-based MASs and establishes the group controllability criteria by adjusting appropriate leaders with switching and fixed topologies, respectively.

3.1. Group Controllability on Switching Topology. Similar to literature [29], corresponding system (5) with switching topology can be described as

$$
\left\{\begin{array}{l}
\dot{x}^{1}=A_{1 \sigma(t)} x^{1}+\left[\begin{array}{ll}
C_{1} & B_{1}
\end{array}\right]_{\sigma(t)}\left[\begin{array}{l}
x^{2} \\
y^{1}
\end{array}\right], \\
\dot{x}^{2}=A_{2 \sigma(t)} x^{2}+\left[\begin{array}{ll}
C_{2} & B_{2}
\end{array}\right]_{\sigma(t)}\left[\begin{array}{l}
x^{1} \\
y^{2}
\end{array}\right],
\end{array}\right.
$$

where the switching path $\sigma(t): \mathbb{R}^{+} \longrightarrow\{1, \ldots, K\}$ can be described by a piecewise constant scalar function, which presents the coupling links of such time-variant system, and $K$ is the number of probable switching topologies. Moreover, we select $\left(A_{i p},\left[C_{i p} B_{i p}\right]\right)(i=1,2, p=1,2, \ldots, K)$ to achieve the system realizations when $\sigma(t)=p$.

Some relevant important concepts will be introduced in the following, and more details can be seen in [29].

Definition 1 (see [29], switching sequence). A finite scalars' set $\pi \triangleq\left\{i_{0}, \ldots, i_{P-1}\right\}$ is said to be a switching sequence, where $P \in(0, \infty)$ indicates the length of $\pi$, and a switching path $\sigma(p)$ is defined as $\sigma(p)=i_{p}$ if $p \in \underline{P}$ $(\underline{P} \triangleq 0,1, \ldots, P-1)$ with $i_{p} \in\{1,2, \ldots, P\}$ be the index of the $p$ th realization.
Definition 2 (group switching controllability). A nonzero state $x$ of system (6) attains group switching controllability if

(1) There are a time instant $P \in(0, \infty)$, a switching path $\sigma: \underline{P} \longrightarrow 1, \ldots, K$, and the input $\left(x^{2}, y^{1}\right)$ for $t \in \underline{P}$ such that $x^{1}(0)=x^{1}$ and $x^{1}(P)=0$

(2) There are a time instant $P \in(0, \infty)$, a switching path $\sigma: \underline{P} \longrightarrow 1, \ldots, K$, and the input $\left(x^{1}, y^{2}\right)$ for $t \in \underline{P}$ such that $x^{2}(0)=x^{2}$ and $x^{2}(P)=0$

Definition 3 (see [29], column space). For a preset matrix $B_{p \times m}=\left[b_{1}, \ldots, b_{m}\right]$, the column space $\mathscr{R}(B)$ is spanned by vectors $b_{1}, b_{2}, \ldots, b_{m}$, denoted as $\mathscr{R}(B) \triangleq \operatorname{span}\left\{b_{1}, \ldots, b_{m}\right\}$.

Lemma 1. (see [29]). For matrices $A_{i} \in R^{p \times m_{i}}$, $i \in \underline{r} \triangleq\{1,2, \ldots, r\} \quad$ and $B=\left[A_{1}, A_{2}, \ldots, A_{r}\right] \in \mathbb{R}^{p \times m}$, $m=\sum_{i=1}^{r} m_{i}$, then $\mathscr{R}(B)=\sum_{i=1}^{r} \mathscr{R}\left(A_{i}\right)$.

Definition 4 (see [29], cyclic invariant subspace). For $A \in R^{N \times N}$ and a linear subspace $\mathscr{W} \subseteq \mathbb{R}^{N},\langle A \mid \mathscr{W}\rangle$ is called as the $\mathscr{R}$-cyclic invariant subspace indicated as $\langle A \mid \mathscr{W}\rangle \triangleq \sum_{i=1}^{K} A^{i-1} \mathscr{W}$.

For notational simplicity, let $\left\langle A_{1} \mid\left(C_{1} B_{1}\right)\right\rangle \triangleq\left\langle A_{1} \mid \mathscr{R}\left(C_{1} B_{1}\right)\right\rangle \triangleq\left\langle A_{1} \mid \mathscr{R}\left(r_{i j}\right)\right\rangle$ for $i=$ 1 and $j=1,2, \ldots, l+n$, and $\left\langle A_{2} \mid\left(C_{2} B_{2}\right)\right\rangle \triangleq\left\langle A_{2} \mid \mathscr{R}\left(r_{i j}\right)\right\rangle$ for $i=2$ and $j=1,2, \ldots, m+k$, where $\left(C_{i} B_{i}\right) \triangleq\left(r_{i j}\right)$. For system (6), the subspace sequence is defined as

$$
\begin{aligned}
\mathscr{W}_{11} & =\sum_{i=1}^{K}\left\langle A_{1 i} \mid r_{1 i}\right\rangle, \mathscr{W}_{12}=\sum_{i=1}^{K}\left\langle A_{1 i} \mid \mathscr{W}_{11}\right\rangle, \ldots, \mathscr{W}_{1 m} \\
& =\sum_{i=1}^{K}\left\langle A_{1 i} \mid \mathscr{W}_{1(m-1)}\right\rangle, \\
\mathscr{W}_{21} & =\sum_{i=1}^{K}\left\langle A_{2 i} \mid r_{2 i}\right\rangle, \mathscr{W}_{22}=\sum_{i=1}^{K}\left\langle A_{2 i} \mid \mathscr{W}_{21}\right\rangle, \ldots, \mathscr{W}_{2 n} \\
& =\sum_{i=1}^{K}\left\langle A_{2 i} \mid \mathscr{W}_{2(n-1)}\right\rangle .
\end{aligned}
$$

Lemma 2. System (6) attains group switching controllability iff $\mathscr{W}_{1 m}=\mathbb{R}^{m}$ and $\mathscr{W}_{2 n}=\mathbb{R}^{n}$.

Proof. Similar proof can be referred from that of Lemma 2 in [29]; here, it is omitted.

Theorem 1. System (6) attains group switching controllability if

$$
\begin{aligned}
& \sum_{i=1}^{K} \mathscr{R}\left(r_{1 i}\right)=\mathbb{R}^{m}, \\
& \sum_{i=1}^{K} \mathscr{R}\left(r_{2 i}\right)=\mathbb{R}^{n} .
\end{aligned}
$$

Proof. Obviously, for $i=1,2, \ldots, K$, 


$$
\begin{aligned}
\mathscr{R}\left(r_{1 i}\right) & \subseteq \mathscr{R}\left(r_{1 i}\right)+\mathscr{R}\left(A_{1 i} r_{1 i}\right)+\cdots+\mathscr{R}\left(A_{1 i}^{m-1} r_{1 i}\right) \\
& =\left\langle A_{1 i} \mid r_{1 i}\right\rangle,
\end{aligned}
$$

we have

$$
\begin{aligned}
\mathbb{R}^{m} & =\mathscr{R}\left(r_{11}\right)+\mathscr{R}\left(r_{12}\right)+\cdots+\mathscr{R}\left(r_{1 K}\right) \\
& \subseteq\left\langle A_{11} \mid r_{11}\right\rangle+\left\langle A_{12} \mid r_{12}\right\rangle+\cdots+\left\langle A_{1 K} \mid r_{1 K}\right\rangle \\
& =\mathscr{W}_{11} \subseteq \mathscr{W}_{12} \subseteq \cdots \subseteq \mathscr{W}_{1 m} .
\end{aligned}
$$

On the contrary, it is easy to know $\mathscr{W}_{1 m} \subseteq \mathbb{R}^{m}$. Therefore, we can have $\mathscr{W}_{1 m}=\mathbb{R}^{m}$. For the subspace $\mathscr{W}_{2 n}$, we can also have the similar result $\mathscr{W}_{2 n}=\mathbb{R}^{n}$. From Lemmas 1 and 2, the assertion holds.

Remark 3. Theorem 1 provides an important and simple method to check the controllability of continuous-time MASs with leaders by designing a switching path. At the same time, it is noted that the group controllability of continuous-time MASs with leaders can depend on leaderto-follower information communications (i.e., matrices $B_{i}$ ) and the subgroup-to-subgroup information communications (i.e., matrices $C_{i}$ ) regardless of the internal information communications between subgroups (i.e., matrices $A_{i}$ ) whether the topology of the internal network is fixed or switching, that is, the controllability of the subgroups is not required, which provides an important convenience for designing a switching path to ensure the group controllability for continuous-time MASs with switching topology.

In the following, some special important cases are discussed.

3.2. Group Controllability on Fixed Topology. When $\sigma(t)=1$, system (6) (equivalently, (5)) presents an MAS based on fixed topology.

Definition 5 (see [30] (group controllability)). A nonzero state $x$ of system (5) attains group controllability if

(1) There are a finite time $T \in J$ and the input $\left(x^{2}, y^{1}\right)$ such that $x^{1}(0)=x^{1}$ and $x^{1}(T)=0$

(2) There are a finite time $T \in J$ and the input $\left(x^{1}, y^{2}\right)$ such that $x^{2}(0)=x^{2}$ and $x^{2}(T)=0$
Lemma 3. System (5) attains group controllability iff $\operatorname{rank}\left(Q_{1}\right)=m$ and $\operatorname{rank}\left(Q_{2}\right)=n$, where

$$
\begin{aligned}
& Q_{1}=\left[\left(C_{1} B_{1}\right) \vdots A_{1}\left(C_{1} B_{1}\right) \vdots A_{1}^{2}\left(C_{1} B_{1}\right) \vdots \cdots \vdots A_{1}^{m-1}\left(C_{1} B_{1}\right)\right], \\
& Q_{2}=\left[\left(C_{2} B_{2}\right) \vdots A_{2}\left(C_{2} B_{2}\right) \vdots A_{2}^{2}\left(C_{2} B_{2}\right) \vdots \cdots \vdots A_{2}^{n-1}\left(C_{2} B_{2}\right)\right] .
\end{aligned}
$$

Here, $Q_{1}$ is the controllability matrix of $\left(\mathscr{G}_{1}, x^{1}\right)$, and $Q_{2}$ is the controllability matrix of $\left(\mathscr{G}_{2}, x^{2}\right)$.

Proof. The result is obvious from Definition 5.

Remark 4. From Lemma 3, it is too complex to compute the controllability matrices of system (5). On this basis, the group controllability of such MAS with leaders is shown by the technique of $\mathrm{PBH}$ rank test.

Theorem 2 (PBH rank test). System (5) attains group controllability iff system (5) satisfies

(1) $\operatorname{rank}\left(s I-A_{1}, C_{1}, B_{1}\right)=m$ and $\operatorname{rank}\left(t I-A_{2}, C_{2}\right.$, $\left.B_{2}\right)=n, \forall s, t \in \mathbb{C}$, where $\mathbb{C}$ is a complex number set

(2) $\operatorname{rank}\left(\lambda_{i} I-A_{1}, C_{1}, B_{1}\right)=m$ and $\operatorname{rank}\left(\mu_{i} I-A_{2}, C_{2}\right.$, $\left.B_{2}\right)=n$, where $\lambda_{i}(\forall i=1, \ldots, m)$ and $\mu_{i}(\forall i=1$, $\ldots, n)$ are, respectively, the eigenvalues of $A_{1}$ and $A_{2}$

Proof. Obviously, if condition (1) holds, condition (2) absolutely holds. Therefore, it is only necessary to prove that condition (1) is true.

Necessity: by contradiction, supposed that $\exists s \in \mathbb{C}$; then,

$$
\operatorname{rank}\left(s I-A_{1}, C_{1}, B_{1}\right)<m,
$$

and then the rows of $\left[s I-A_{1}, C_{1}, B_{1}\right]$ are linearly dependent. Thus, $\exists \alpha(\neq 0)$ such that $\alpha^{\prime}\left[s I-A_{1}, C_{1}, B_{1}\right]=0$. Therefore,

$$
\begin{aligned}
s \alpha^{\prime} & =\alpha / A_{1}, \\
\alpha^{\prime} C_{1} & =0, \\
\alpha^{\prime} B_{1} & =0 .
\end{aligned}
$$

Moreover, we can have

$$
\begin{aligned}
& \alpha^{\prime} Q_{1}=\alpha^{\prime}\left[C_{1} A_{1} C_{1} A_{1}^{2} C_{1} \cdots A_{1}^{m-1} C_{1} \vdots B_{1} A_{1} B_{1} A_{1}^{2} B_{1} \cdots A_{1}^{m-1} B_{1}\right] \\
& =\left[\alpha^{\prime} C_{1} \alpha^{\prime} A_{1} C_{1} \alpha^{\prime} A_{1}^{2} C_{1} \cdots \alpha^{\prime} A_{1}^{m-1} C_{1} \vdots \alpha^{\prime} B_{1} \alpha^{\prime} A_{1} B_{1} \alpha^{\prime} A_{1}^{2} B_{1} \cdots \alpha^{\prime} A_{1}^{m-1} B_{1}\right] \\
& =\left[\alpha^{\prime} B_{1} s \alpha^{\prime} B_{1} \cdots s^{m-1} \alpha^{\prime} B_{1}: \alpha^{\prime} C_{1} s \alpha^{\prime} C_{1} \cdots s^{m-1} \alpha^{\prime} C_{1}\right]=0 \text {. }
\end{aligned}
$$

Since $\alpha \neq 0$, then there must be $\operatorname{rank}\left(Q_{1}\right)<m$, which implies that system (5) is uncontrollable, contradicting to the assertion that system (5) attains the group controllability. The necessity of (1) is proved. 
Sufficiency: by contradiction, assumed that system (5) is uncontrollable, then $\exists \lambda \in \mathbb{C}$ of $A_{1}$, which corresponds to the eigenvector $\beta(\neq 0)$ satisfying

$$
\begin{aligned}
& \beta^{\prime} A_{1}=\lambda \beta^{\prime}, \\
& \beta^{\prime} C_{1}=0, \\
& \beta^{\prime} B_{1}=0,
\end{aligned}
$$

and then $\beta^{\prime}\left[\lambda I-A_{1}, C_{1}, B_{1}\right]=0$ so that $\operatorname{rank}(\lambda I-$ $\left.A_{1}, C_{1}, B_{1}\right)<m$. This contradicts to $\operatorname{rank}\left(s I-A_{1}, C_{1}, B_{1}\right)=$ $m$ for $\forall s \in \mathbb{C}$. The sufficiency of (1) is proved.
(1) The eigenvalues of $A_{i}$ are different

(2) The eigenvectors of $A_{i}$ are unorthogonal to at least one column of $B_{i}$ or $C_{i}$

Proof. Since $L_{1}^{T}=L_{1}$, then $A_{1}^{T}=A_{1}$, which can be displayed as $A_{1}=U_{1} \Lambda_{1} U_{1}^{T}$, where the columns of $U_{1}$ and the diagonal matrix $\Lambda_{1}$ are made up of orthogonal eigenvectors and eigenvalues of $A_{1}$, respectively. Moreover, $U_{1} U_{1}{ }^{T}=I$; then, the controllability matrix of such a system can be expressed as

Theorem 3. If $L_{i}^{T}=L_{i}(i=1,2)$, system (5) attains group controllability iff

$$
\begin{aligned}
& Q_{1}=\left[\begin{array}{llllllll}
B_{1} & A_{1} B_{1} & A_{1}^{2} B_{1} & \cdots & A_{1}^{m-1} B_{1} & \vdots C_{1} & A_{1} C_{1} & A_{1}^{2} C_{1} \cdots A_{1}^{m-1} C_{1}
\end{array}\right] \\
& =\left[U_{1} U_{1}^{T} B_{1} \quad U_{1} \Lambda_{1} U_{1}^{T} B_{1} \quad U_{1} \Lambda_{1}^{2} U_{1}^{T} B_{1} \cdots U_{1} \Lambda_{1}^{m-1} U_{1}^{T} B_{1} \vdots U_{1} U_{1}^{T} C_{1} \quad U_{1} \Lambda_{1} U_{1}^{T} C_{1} \cdots U_{1} \Lambda_{1}^{m-1} U_{1}^{T} C_{1}\right] \\
& =U_{1}\left[U_{1}^{T} B_{1} \quad \Lambda_{1} U_{1}^{T} B_{1} \quad \Lambda_{1}^{2} U_{1}^{T} B_{1} \cdots \Lambda_{1}^{m-1} U_{1}^{T} B_{1} \vdots U_{1}^{T} C_{1} \quad \Lambda_{1} U_{1}^{T} C_{1} \quad \Lambda_{1}^{2} U_{1}^{T} C_{1} \cdots \Lambda_{1}^{m-1} U_{1}^{T} C_{1}\right] \\
& \triangleq U_{1} \widetilde{Q_{1}}
\end{aligned}
$$

where $\widetilde{Q_{1}}=\left[U_{1}^{T} B_{1} \Lambda_{1} U_{1}^{T} B_{1} \Lambda_{1}^{2} U_{1}^{T} B_{1} \cdots \Lambda_{1}^{m-1} U_{1}^{T} \quad B_{1}: U_{1}^{T} C_{1} \Lambda_{1}\right.$ $\left.U_{1}^{T} C_{1} \Lambda_{1}^{2} U_{1}^{T} C_{1} \cdots \Lambda_{1}^{m-1} U_{1}^{T} C_{1}\right]$

Since $U_{1}$ consists of the orthogonal eigenvectors of $A_{1}$, then $U_{1}$ is nonsingular, which implies that $\operatorname{rank}\left(Q_{1}\right)=\operatorname{rank}\left(\widetilde{Q_{1}}\right)$. Let $\lambda_{i}$ and $\eta_{i}$ be the eigenvalues and their corresponding eigenvectors of $A_{1}$, respectively, for $i=1,2, \ldots, m$.
For the convenience of discussion, let $U_{1}^{T} B_{1} \triangleq\left[r_{1}, \ldots, r_{l}\right], U_{1}^{T} C_{1} \triangleq\left[p_{1}, \ldots, p_{n}\right], r_{i} \triangleq\left[r_{1 i}, \ldots, r_{m i}\right]^{T}$ with $r_{k i}=\left(\eta_{k}, b_{i}\right),(k=1,2, \ldots, m ; i=1,2, \ldots, l)$, and $p_{i}=\left[p_{1 i}\right.$, $\left.p_{2 i}, \ldots, p_{m i}\right]^{T}(i=1,2, \ldots, n)$; then, 


$$
\begin{aligned}
& \widetilde{Q_{1}}=\left\{\left(r_{1}, r_{2}, \ldots, r_{l}\right),\left[\begin{array}{llll}
\lambda_{1} & & & \\
& \lambda_{2} & & \\
& & \ddots & \\
& & & \lambda_{m}
\end{array}\right]\left(r_{1}, r_{2}, \ldots, r_{l}\right), \ldots,\left[\begin{array}{llll}
\lambda_{1}^{m-1} & & & \\
& \lambda_{2}^{m-1} & & \\
& & \ddots & \\
& & & \lambda_{m}^{m-1}
\end{array}\right]\left(r_{1}, r_{2}, \ldots, r_{l}\right) \vdots\right. \\
& \left.\left(p_{1}, p_{2}, \ldots, p_{n}\right),\left[\begin{array}{cccc}
\lambda_{1} & & & \\
& \lambda_{2} & & \\
& & \ddots & \\
& & & \lambda_{m}
\end{array}\right]\left(p_{1}, p_{2}, \ldots, p_{n}\right), \ldots,\left[\begin{array}{cccc}
\lambda_{1}^{m-1} & & & \\
& \lambda_{2}^{m-1} & & \\
& & \ddots & \\
& & & \lambda_{m}^{m-1}
\end{array}\right]\left(p_{1}, p_{2}, \ldots, p_{n}\right)\right\} \\
& \longrightarrow\left\{\left(r_{1}, \Lambda_{1} r_{1}, \cdots, \Lambda_{1}^{m-1} r_{1}\right), \ldots,\left(r_{l}, \Lambda_{1} r_{l}, \ldots, \Lambda_{1}^{m-1} r_{l}\right) \vdots\left(p_{1}, \Lambda_{1} p_{1}, \ldots, \Lambda_{1}^{m-1} p_{1}\right), \ldots,\left(p_{n}, \Lambda_{1} p_{n}, \ldots, \Lambda_{1}^{m-1} p_{n}\right)\right\} \\
& \longrightarrow\left\{\left[\begin{array}{ccccc}
r_{11} & \lambda_{1} r_{11} & \lambda_{1}^{2} r_{11} & \cdots & \lambda_{1}^{m-1} r_{11} \\
r_{21} & \lambda_{2} r_{21} & \lambda_{2}^{2} r_{21} & \ldots & \lambda_{2}^{m-1} r_{21} \\
\vdots & \vdots & \vdots & & \vdots \\
r_{m 1} & \lambda_{m} r_{m 1} & \lambda_{m}^{2} r_{m 1} & \ldots & \lambda_{m}^{m-1} r_{m 1}
\end{array}\right], \ldots,\left[\begin{array}{ccccc}
r_{1 l} & \lambda_{1} r_{1 l} & \lambda_{1}^{2} r_{1 l} & \ldots & \lambda_{1}^{m-1} r_{1 l} \\
r_{2 l} & \lambda_{2} r_{2 l} & \lambda_{2}^{2} r_{2 l} & \ldots & \lambda_{2}^{m-1} r_{2 l} \\
\vdots & \vdots & \vdots & & \vdots \\
r_{m l} & \lambda_{m} r_{m l} & \lambda_{m}^{2} r_{m l} & \ldots & \lambda_{m}^{m-1} r_{m l}
\end{array}\right] \vdots\right. \\
& \left.\left[\begin{array}{ccccc}
p_{11} & \lambda_{1} p_{11} & \lambda_{1}^{2} p_{11} & \cdots & \lambda_{1}^{m-1} p_{11} \\
p_{21} & \lambda_{2} p_{21} & \lambda_{2}^{2} p_{21} & \cdots & \lambda_{2}^{m-1} p_{21} \\
\vdots & \vdots & \vdots & & \vdots \\
p_{m 1} & \lambda_{m} p_{m 1} & \lambda_{m}^{2} p_{m 1} & \cdots & \lambda_{m}^{m-1} p_{m 1}
\end{array}\right], \ldots,\left[\begin{array}{ccccc}
p_{1 n} & \lambda_{1} p_{1 n} & \lambda_{1}^{2} p_{1 n} & \cdots & \lambda_{1}^{m-1} p_{1 n} \\
p_{2 n} & \lambda_{2} p_{2 n} & \lambda_{2}^{2} p_{2 n} & \cdots & \lambda_{2}^{m-1} p_{2 n} \\
\vdots & \vdots & \vdots & & \vdots \\
p_{m n} & \lambda_{m} p_{m n} & \lambda_{m}^{2} p_{m n} & \cdots & \lambda_{m}^{m-1} p_{m n}
\end{array}\right]\right\} \\
& \longrightarrow\left\{\left[\begin{array}{cccc}
r_{11} & & & \\
& r_{21} & & \\
& & \ddots & \\
& & & r_{m 1}
\end{array}\right]\left[\begin{array}{cccc}
1 & \lambda_{1} & \cdots & \lambda_{1}^{m-1} \\
1 & \lambda_{2} & \cdots & \lambda_{2}^{m-1} \\
\vdots & \vdots & & \vdots \\
1 & \lambda_{m} & \cdots & \lambda_{m}^{m-1}
\end{array}\right], \cdots,\left[\begin{array}{cccc}
r_{1 l} & & & \\
& r_{2 l} & & \\
& & \ddots & \\
& & & r_{m l}
\end{array}\right]\left[\begin{array}{cccc}
1 & \lambda_{1} & \cdots & \lambda_{1}^{m-1} \\
1 & \lambda_{2} & \cdots & \lambda_{2}^{m-1} \\
\vdots & \vdots & & \vdots \\
1 & \lambda_{m} & \cdots & \lambda_{m}^{m-1}
\end{array}\right] \vdots\right. \\
& \left.\left[\begin{array}{cccc}
p_{11} & & & \\
& p_{21} & & \\
& & \ddots & \\
& & & p_{m 1}
\end{array}\right]\left[\begin{array}{cccc}
1 & \lambda_{1} & \cdots & \lambda_{1}^{m-1} \\
1 & \lambda_{2} & \cdots & \lambda_{2}^{m-1} \\
\vdots & \vdots & & \vdots \\
1 & \lambda_{m} & \cdots & \lambda_{m}^{m-1}
\end{array}\right], \ldots,\left[\begin{array}{cccc}
p_{1 n} & & & \\
& p_{2 n} & & \\
& & \ddots & \\
& & & p_{m n}
\end{array}\right]\left[\begin{array}{cccc}
1 & \lambda_{1} & \cdots & \lambda_{1}^{m-1} \\
1 & \lambda_{2} & \cdots & \lambda_{2}^{m-1} \\
\vdots & \vdots & & \vdots \\
1 & \lambda_{m} & \cdots & \lambda_{m}^{m-1}
\end{array}\right]\right\} \\
& \longrightarrow\left\{\left[\begin{array}{llll}
\left(\eta_{1}, b_{1}\right) & & & \\
& \left(\eta_{2}, b_{1}\right) & & \\
& & \ddots & \\
& & & \left(\eta_{m}, b_{1}\right)
\end{array}\right] M, \ldots,\left[\begin{array}{llll}
\left(\eta_{1}, b_{l}\right) & & & \\
& \left(\eta_{2}, b_{l}\right) & & \\
& \ddots & \\
& & \left(\eta_{m}, b_{l}\right)
\end{array}\right] M, \ldots\right.
\end{aligned}
$$

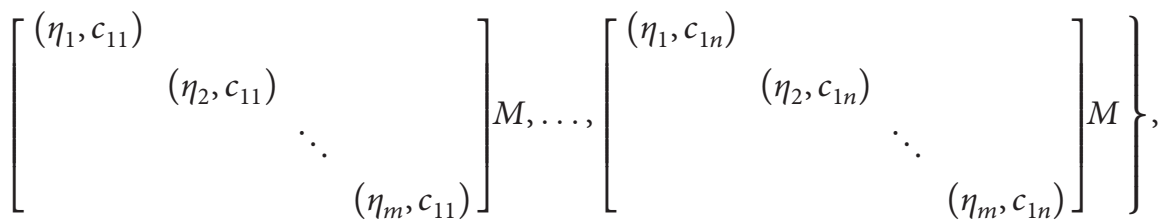

where $\left(\eta_{1}, b_{l}\right),\left(\eta_{m}, c_{1 n}\right)$ is the vector inner product and matrix

$$
M=\left[\begin{array}{ccccc}
1 & \lambda_{1} & \lambda_{1}^{2} & \cdots & \lambda_{1}^{m-1} \\
1 & \lambda_{2} & \lambda_{2}^{2} & \cdots & \lambda_{2}^{m-1} \\
\vdots & \vdots & \vdots & & \vdots \\
1 & \lambda_{m} & \lambda_{m}^{2} & \cdots & \lambda_{m}^{m-1}
\end{array}\right],
$$


is a Vandermonde matrix. If $\widetilde{Q}_{1}$ has full row rank needing that at least one block of $\widetilde{Q}_{1}$ has full row rank, without loss of generality, the first block will be selected to discuss. It is known that Vandermonde matrix $M$ is nonsingular if eigenvalues of $A_{1}$ are distinct so that the row rank of matrix $\widetilde{Q_{1}}$ is decided by matrix $\operatorname{diag}\left\{\left(\eta_{1}, b_{1}\right),\left(\eta_{2}, b_{1}\right), \ldots,\left(\eta_{m}, b_{1}\right)\right\}$ or matrix $\operatorname{diag}\left\{\left(\eta_{1}, c_{11}\right),\left(\eta_{2}, c_{11}\right), \ldots,\left(\eta_{m}, c_{11}\right)\right\}$. Since eigenvectors $A_{i}$ are unorthogonal to at least one column of $B_{i}$ or $C_{i}(i=1,2)$, therefore, matrix $\operatorname{diag}\left\{\left(\eta_{1}, b_{1}\right)\right.$, $\left.\left(\eta_{2}, b_{1}\right), \ldots,\left(\eta_{m}, b_{1}\right)\right\}$ or matrix $\operatorname{diag}\left\{\left(\eta_{1}, c_{11}\right),\left(\eta_{2}, c_{11}\right), \ldots\right.$, $\left.\left(\eta_{m}, c_{11}\right)\right\}$ has full row rank, which means that $\widetilde{Q}_{1}$ has full row rank. Similarly, $\widetilde{Q}_{2}$ also has full row rank. This completes the proof.

Note that condition $L_{i}^{T}=L_{i}$ implies that the information weight from agent $i$ to agent $j$ is the same as that from agent $j$ to agent $i$ in the same subgroup, that is, the topological structure is symmetric for the subgroups.

Corollary 1. System (5) is uncontrollable if subgroups $\left(\mathscr{G}, x^{1}\right)$ and $\left(\mathscr{G}, x^{2}\right)$ are both complete graphs (see Figure 2) and $a_{i j}=b_{i q}\left(\forall i, j \in \ell_{1}, \ell_{2}, \forall q \in \ell_{l}\right)$, regardless of how to connect $\left(\mathscr{G}, x^{1}\right)$ and $\left(\mathscr{G}, x^{2}\right)$.

Proof. Because subgraphs $\left(\mathscr{G}, x^{1}\right)$ and $\left(\mathscr{G}, x^{2}\right)$ are both complete and $a_{i j}=b_{i q}\left(\forall i, j \in \ell_{1}, \ell_{2}, \forall q \in \ell_{l}\right)$, without loss of generality, let $a_{i j}=b_{i q}=1\left(\forall i, j \in \ell_{1}, \ell_{2}, \forall q \in \ell_{l}\right)$; then,

$$
\begin{aligned}
& A_{1}=\left[\begin{array}{cccc}
-(m+l-1) & 1 & \cdots & 1 \\
1 & -(m+l-1) & \cdots & 1 \\
\vdots & \vdots & & \vdots \\
1 & 1 & \cdots & -(m+l-1)
\end{array}\right], \\
& A_{2}=\left[\begin{array}{cccc}
-(n+k-1) & 1 & \cdots & 1 \\
1 & -(n+k-1) & \cdots & 1 \\
\vdots & \vdots & & \vdots \\
1 & 1 & \cdots & -(n+k-1)
\end{array}\right] .
\end{aligned}
$$

By simple calculation, we can know that $A_{1}$ 's eigenvalues are $\lambda_{i}=\underbrace{0,-(m+l), \ldots,-(m+l)}$, and $A_{2}$ 's eigenvalues are $\mu_{i}=\underbrace{0,-(n+k), \ldots,-(n+k)}$. Then, $A_{1}$ has common eigenvalue - $(m+l)$, and $A_{2}$ has common eigenvalue - $(n+k)$, which are contrary to the conditions of Theorem 3 . Thus, no matter how to connect subgroups $\left(\mathscr{G}_{1}, x^{1}\right)$ and $\left(\mathscr{G}_{2}, x^{2}\right)$, system (5) is uncontrollable.

Corollary 2. If $\left(\mathscr{G}, x^{1}\right)$ and $\left(\mathscr{G}, x^{2}\right)$ are both star graphs (see Figure 3) as well as $a_{i j}=b_{i q}\left(\forall i, j \in \ell_{1}, \ell_{2}, \forall q \in \ell_{l}\right)$ regardless of how to connect $\left(\mathscr{G}, x^{1}\right)$ and $\left(\mathscr{G}, x^{2}\right)$, then system (5) is uncontrollable.

Proof. Because subgraphs $\left(\mathscr{G}, x^{1}\right)$ and $\left(\mathscr{G}, x^{2}\right)$ are both star groups and $a_{i j}=b_{i q}\left(\forall i, j \in \ell_{1}, \ell_{2}, \forall q \in \ell_{l}\right)$, without loss of generality, let $a_{i j}=b_{i q}=1\left(\forall i, j \in \ell_{1}, \ell_{2}, \forall q \in \ell_{l}\right)$; therefore,

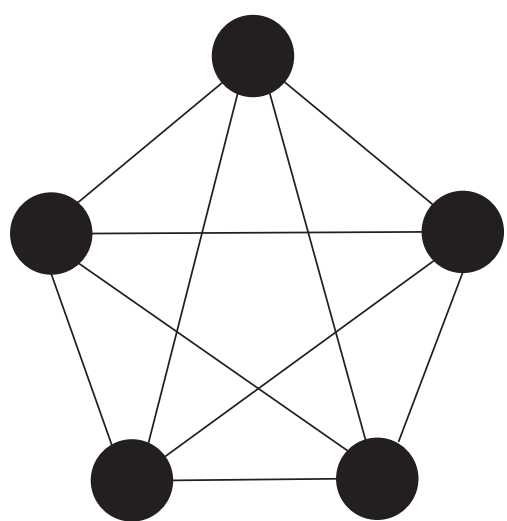

FIgURE 2: Complete graph.

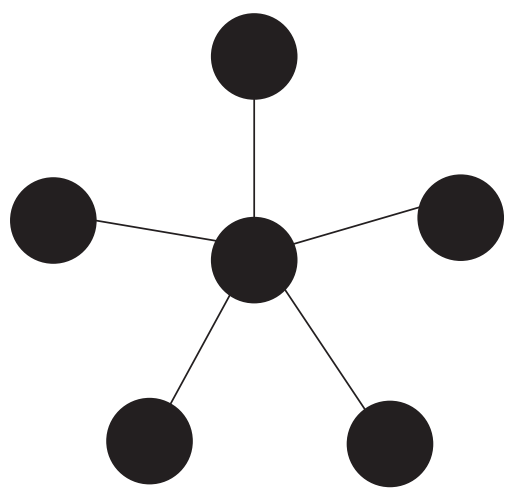

Figure 3: Star graph.

$$
\begin{aligned}
A_{1} & =\left[\begin{array}{cccc}
-m-l-1 & 1 & \cdots & 1 \\
1 & -m-l-1 & \cdots & 1 \\
\vdots & \vdots & & \vdots \\
1 & 1 & \cdots & -m-l-1
\end{array}\right], \\
A_{2} & =\left[\begin{array}{cccc}
-n-k-1 & 1 & \cdots & 1 \\
1 & -n-k-1 & \cdots & 1 \\
\vdots & \vdots & & \vdots \\
1 & 1 & \cdots & -n-k-1
\end{array}\right] .
\end{aligned}
$$

By computing, we can also know that the eigenvalues of $A_{1}$ are $\lambda_{i}=\underbrace{0,-(m+l+2), \ldots,-(m+l+2)}$, and the eigenvalues of $A_{2}$ are $\mu_{i}=\underbrace{0,-(n+k+2), \ldots,-(n+k+2)}$. Then, $A_{1}$ has common eigenvalues $-(m+l+2)$, and $A_{2}$ has common eigenvalues $-(n+k+2)$, which contradict to condition (1) of Theorem 3. Thus, no matter how to connect subgroup $\left(\mathscr{G}_{1}, x^{1}\right)$ and subgroup $\left(\mathscr{G}_{2}, x^{2}\right)$, system (5) must be uncontrollable.

Remark 5. It is noted that there must exist a few leaders making the system to reach the desired state from the random initial state if system (5) is controllable. However, how to configure the leaders such that the desired formation can be achieved? That is, how to select the leaders (or design the inputs) with given initial state and desired state?

Here presents an algorithm for designing the leaders. 
Algorithm 1 (algorithm for designing leaders). For the given initial and desired states $x(0)$ and $x\left(t_{1}\right)$, MASs can reach the desired state during $\left[0, t_{1}\right]$, where $t_{1}>0$ is the finial time. Suppose that MAS (5) is controllable; then, its Gram matrix is

$$
W_{c}\left[0, t_{1}\right] \triangleq \int_{0}^{t_{1}} e^{-A t}[C B][C B]^{T} e^{-A^{T} t} \mathrm{~d} t,
$$

where $t \in\left[0, t_{1}\right]$. Since $W_{c}\left[0, t_{1}\right]$ is invertible, we can design a set of inputs (leaders) as

$$
u(t)=-[C B]^{T} e^{-A^{T} t} W_{c}^{-1}\left[0, t_{1}\right] x(0) .
$$

Then, a solution of system (5) is

$$
x\left(t_{1}\right)=e^{A t_{1}} x(0)+\int_{0}^{t_{1}} e^{A\left(t_{1}-t\right)}[C B] u(t) \mathrm{d} t,
$$

which can make the system state from $x(0)$ to $x\left(t_{1}\right)$ during $\left[0, t_{1}\right]$. Notice that $t_{1}$ is the longest time to get a set of inputs.

\section{Example and Simulations}

A nine-agent system with followers 4 and a leader as subgroup 1 and followers 3 and a leader as subgroup 2 is described by Figure 4 with $a_{12}=a_{21}=1, a_{23}=a_{32}=2, a_{34}=a_{43}=$ $1, a_{45}=a_{54}=1, a_{56}=a_{65}=1, a_{67}=a_{76}=1$; otherwise, $a_{i j}=0$.

From Figure 4, the system matrices are as follows:

$$
\begin{aligned}
A_{1} & =\left[\begin{array}{cccc}
-1 & 1 & 0 & 0 \\
1 & -3 & 2 & 0 \\
0 & 2 & -4 & 1 \\
0 & 0 & 1 & -1
\end{array}\right], \\
B_{1} & =\left[\begin{array}{l}
0 \\
0 \\
1 \\
0
\end{array}\right], \\
C_{1} & =\left[\begin{array}{lll}
0 & 0 & 0 \\
0 & 0 & 0 \\
0 & 0 & 0 \\
1 & 0 & 0
\end{array}\right] ; \\
A_{2} & =\left[\begin{array}{ccc}
-2 & 2 & 0 \\
2 & -3 & 1 \\
0 & 1 & 2
\end{array}\right], \\
B_{2} & =\left[\begin{array}{l}
0 \\
0 \\
1
\end{array}\right],
\end{aligned}
$$

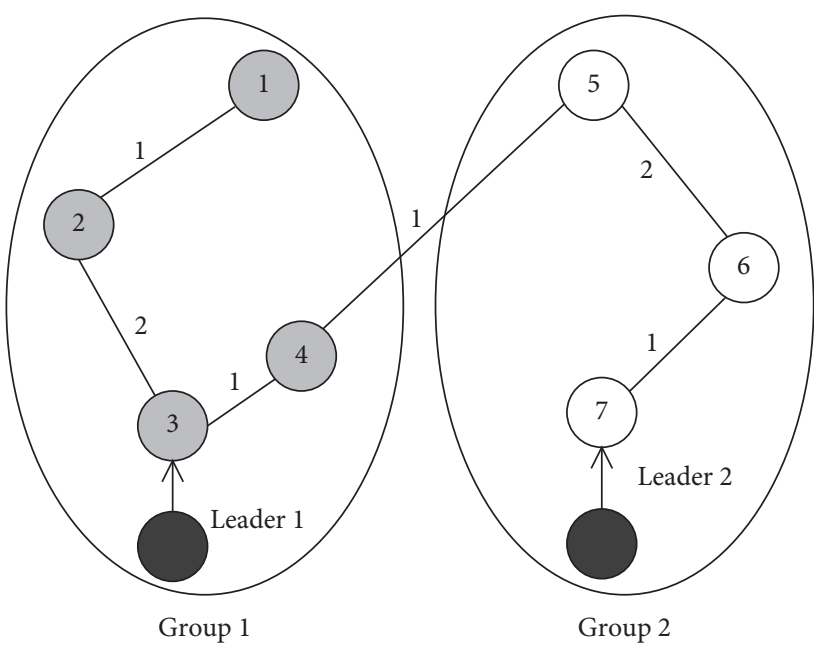

Figure 4: Topology with 9 agents.

By computing, we can get the eigenvalues of $A_{1}$ and $A_{2}$ are $\{-5.7711,-2.2430,-0.7904,-0.1955\}$ and $\{-4.6562$, $-0.5770,2.2332\}$, respectively, and the corresponding eigenvectors are

$$
\begin{aligned}
& \eta_{1}=\left[\begin{array}{c}
0.1262 \\
-0.6023 \\
0.7714 \\
-0.1617
\end{array}\right], \\
& \eta_{2}=\left[\begin{array}{c}
0.4941 \\
-0.6141 \\
-0.4795 \\
0.3858
\end{array}\right], \\
& \eta_{3}=\left[\begin{array}{c}
-0.6023 \\
-0.1262 \\
0.1617 \\
0.7714
\end{array}\right],
\end{aligned}
$$$$
\eta_{4}=\left[\begin{array}{l}
-0.6141 \\
-0.4941 \\
-0.3858 \\
-0.4795
\end{array}\right] \text {; }
$$$$
\mu_{1}=\left[\begin{array}{c}
0.5972 \\
-0.7932 \\
0.1192
\end{array}\right] \text {, }
$$

$\mu_{2}=\left[\begin{array}{c}0.7949 \\ 0.5656 \\ -0.2195\end{array}\right]$,

$\mu_{3}=\left[\begin{array}{l}-0.1067 \\ -0.2258 \\ -0.9683\end{array}\right]$. 


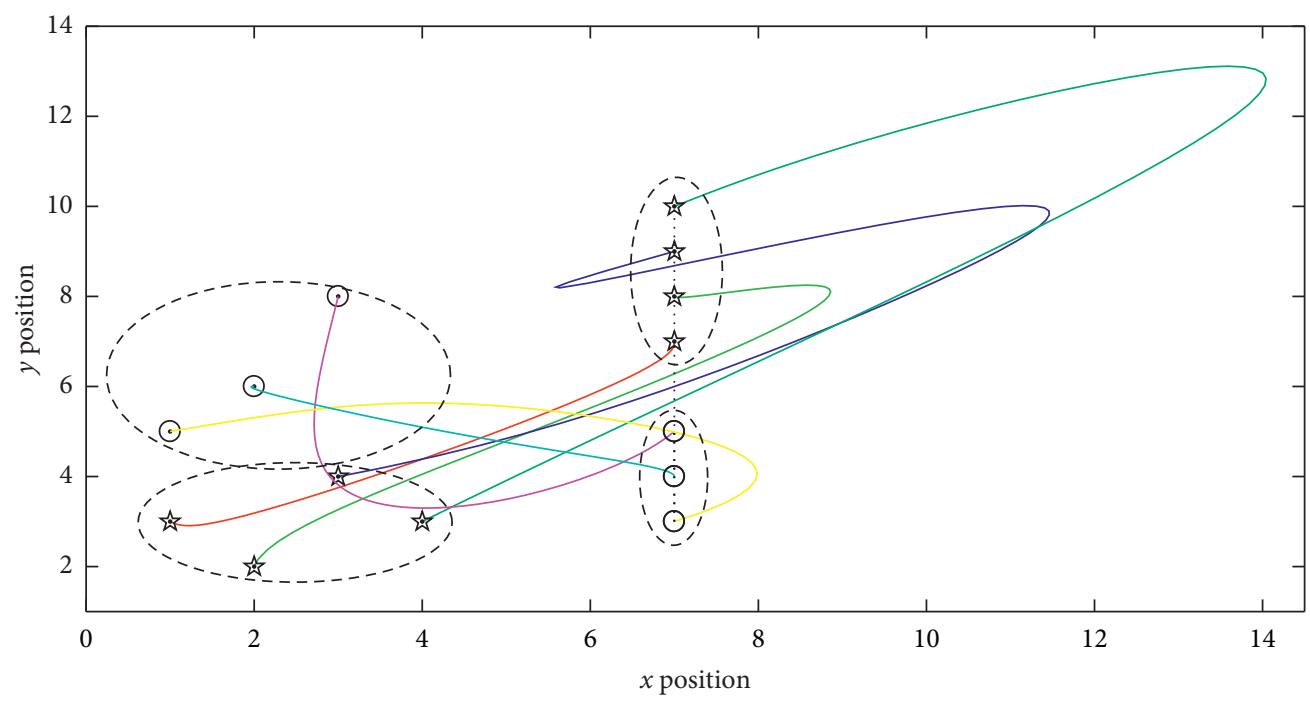

Figure 5: A straight line.

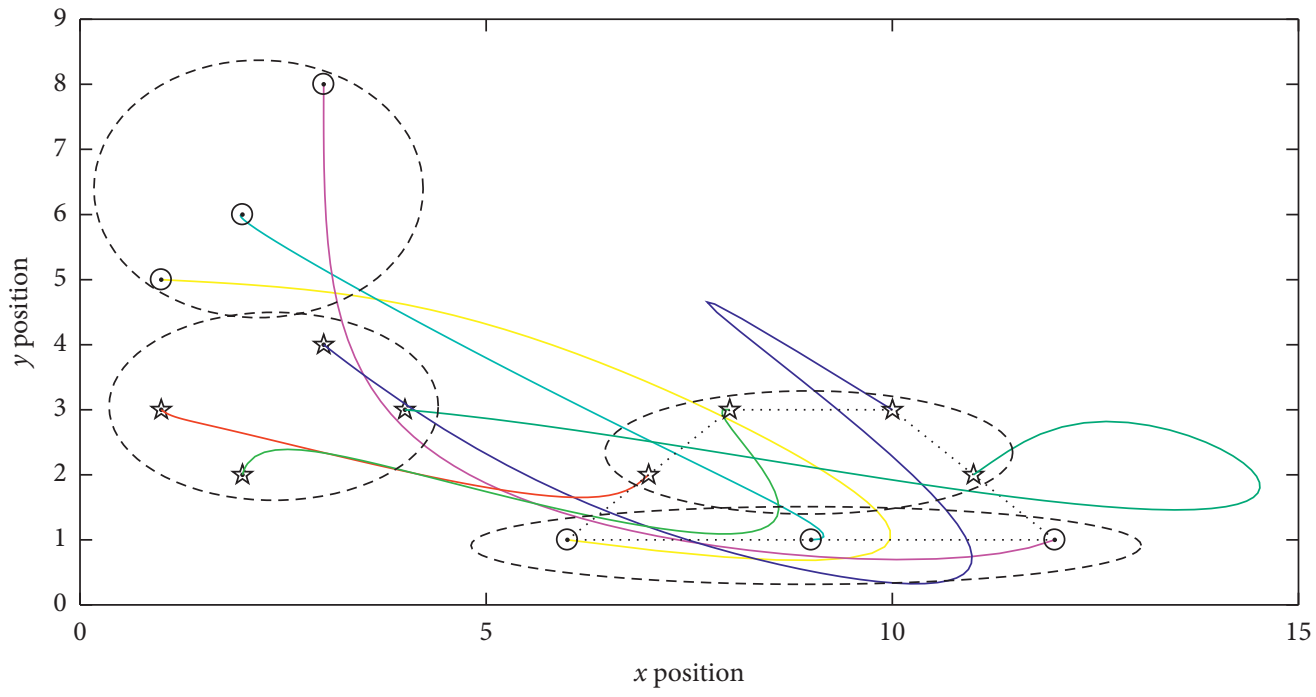

FIgURE 6: A trapezoid.

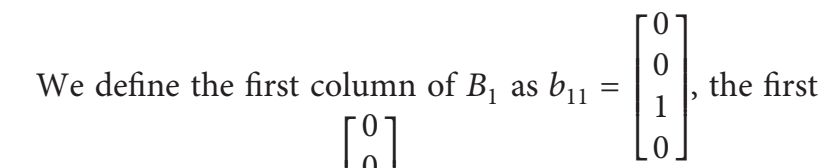
column of $C_{1}$ as $c_{11}=\left[\begin{array}{l}0 \\ 0 \\ 0 \\ 1\end{array}\right]$, the first column of $B_{2}$ as $b_{21}=\left[\begin{array}{l}0 \\ 0 \\ 1\end{array}\right]$, and the last column of $C_{2}$ as $c_{24}=\left[\begin{array}{l}1 \\ 0 \\ 0\end{array}\right]$, as well as calculate the inner products of every eigenvalue and the corresponding vectors as

$$
\begin{aligned}
& \left(\eta_{1}, b_{11}\right)=0.7714, \\
& \left(\eta_{2}, b_{11}\right)=-0.4795, \\
& \left(\eta_{3}, b_{11}\right)=0.1617, \\
& \left(\eta_{4}, b_{11}\right)=-0.3858 \\
& \left(\eta_{1}, c_{11}\right)=-0.1617 \\
& \left(\eta_{2}, c_{11}\right)=0.3858, \\
& \left(\eta_{1}, c_{11}\right)=0.7714, \\
& \left(\eta_{1}, c_{11}\right)=-0.4795,
\end{aligned}
$$


which mean all the eigenvectors of $A_{1}$ are unorthogonal to the one column of $B_{1}$ or $C_{1}$. At the same time,

$$
\begin{aligned}
& \left(\mu_{1}, b_{21}\right)=0.1192, \\
& \left(\mu_{2}, b_{21}\right)=-0.2195, \\
& \left(\mu_{3}, b_{21}\right)=-0.9683, \\
& \left(\mu_{1}, c_{24}\right)=0.5972, \\
& \left(\mu_{2}, c_{24}\right)=0.7949, \\
& \left(\mu_{3}, c_{24}\right)=-0.1067,
\end{aligned}
$$

which mean all the eigenvectors of $A_{2}$ are unorthogonal to any one column of $B_{2}$ or $C_{2}$. Those imply that system (5) described by Figure 4 can attain the group controllability.

Figures 5 and 6 depict the initial states, final states, and moving trajectories of the followers of subgroup 1 and subgroup 2 described by the black star dots and the black circular dots, respectively. Beginning from random initial states, the followers of subgroup 1 and subgroup 2 can be finally governed to a straight-line alignment and a trapezoid alignment, respectively.

\section{Conclusion}

This paper has discussed the group controllability of continuous-time MASs with multiple leaders on switching and fixed topologies, respectively. Some useful and effective results of group controllability are obtained by the rank test and the PBH test. Specially, the group controllability of continuous-time MASs for some special topology graphs has also been studied.

\section{Data Availability}

In this paper, no data are needed; only mathematical derivation is needed.

\section{Conflicts of Interest}

The authors declare that there are no conflicts of interest regarding the publication of this paper.

\section{Acknowledgments}

This work was supported by the National Natural Science Foundation of China under Grant nos. 61773023, 61991412, 61773416, and 61873318, the Program for HUST Academic Frontier Youth Team under Grant no. 2018QYTD07, and the Frontier Research Funds of Applied Foundation of Wuhan under Grant no. 2019010701011421.

\section{References}

[1] X. Wang and H. Su, "Consensus of hybrid multi-agent systems by event-triggered/self-triggered strategy," Applied Mathematics and Computation, vol. 359, pp. 490-501, 2019.

[2] Y. Guan, L. Tian, and L. Wang, "Controllability of switching signed networks," IEEE Transactions on Circuits and Systems II: Express Briefs, 2019.
[3] X. Liu, Z. Ji, and T. Hou, "Graph partitions and the controllability of directed signed networks," Science China Information Sciences, vol. 62, no. 4, Article ID 042202, 2019.

[4] Y. Guan and L. Wang, "Target controllability of multiagent systems under fixed and switching topologies," International Journal of Robust and Nonlinear Control, vol. 29, no. 9, pp. 2725-2741, 2019.

[5] X. Liu, Z. Ji, T. Hou, and H. Yu, "Decentralized stabilizability and formation control of multi-agent systems with antagonistic interactions," ISA Transactions, vol. 89, pp. 58-66, 2019.

[6] Y. Liu and H. Su, "Containment control of second-order multi-agent systems via intermittent sampled position data communication," Applied Mathematics and Computation, vol. 362, Article ID 124522, 2019.

[7] B. Liu, N. Xu, H. Su, L. Wu, and J. Bai, "On the observability of leader-based multiagent systems with fixed topology," Complexity, vol. 2019, Article ID 9487574, 10 pages, 2019.

[8] Y. Sun, Z. Ji, Q. Qi, and H. Ma, "Bipartite consensus of multiagent systems with intermittent interaction," IEEE Access, vol. 7, pp. 130300-130311, 2019.

[9] J. Qu, Z. Ji, C. Lin, and H. Yu, "Fast consensus seeking on networks with antagonistic interactions," Complexity, vol. 2018, Article ID 7831317, 15 pages, 2018.

[10] Z. Ji, H. Lin, S. Cao, Q. Qi, and H. Ma, "The complexity in complete graphic characterizations of multiagent controllability," IEEE Transactions on Cybernetics, 2020.

[11] X. Wang and H. Su, "Self-triggered leader-following consensus of multi-agent systems with input time delay," $\mathrm{Neu}$ rocomputing, vol. 330, pp. 70-77, 2019.

[12] Y. Liu and H. Su, "Some necessary and sufficient conditions for containment of second-order multi-agent systems with sampled position data," Neurocomputing, vol. 378, pp. 228-237, 2020.

[13] Z. Lu, L. Zhang, and L. Wang, "Observability of multi-agent systems with switching topology," IEEE Transactions on Circuits and Systems II: Express Briefs, vol. 64, no. 11, pp. 1317-1321, 2017.

[14] H. Su, J. Zhang, and X. Chen, "A stochastic sampling mechanism for time-varying formation of multiagent systems with multiple leaders and communication delays," IEEE Transactions on Neural Networks and Learning Systems, vol. 30, no. 12, pp. 3699-3707, 2019.

[15] H. G. Tanner, "On the controllability of nearest neighbor interconnections," in Proceedings of the 43rd IEEE Conference on Decision and Control, vol. 1, pp. 2467-2472, Nassau, Bahamas, December 2004.

[16] B. Liu, T. Chu, L. Wang, and G. Xie, "Controllability of a leaderfollower dynamic network with switching topology," IEEE Transactions on Automatic Control, vol. 53, no. 4, pp. 10091013, 2008.

[17] B. Liu, H. Su, L. Wu, and S. He, "A new perspective to algebraic characterization on controllability of multiagent systems," Complexity, vol. 2020, Article ID 9703972, 12 pages, 2020.

[18] Z. Ji, Z. Wang, H. Lin, and Z. Wang, "Interconnection topologies for multi-agent coordination under leader-follower framework," Automatica, vol. 45, no. 12, pp. 2857-2863, 2009.

[19] M. Long, H. Su, and B. Liu, "Second-order controllability of two-time-scale multi-agent systems," Applied Mathematics and Computation, vol. 343, pp. 299-313, 2019.

[20] X. Liu and Z. Ji, "Controllability of multiagent systems based on path and cycle graphs," International Journal of Robust and Nonlinear Control, vol. 28, no. 1, pp. 296-309, 2018.

[21] M. Long, H. Su, and B. Liu, "Group controllability of twotime-scale multi-agent networks," Journal of the Franklin Institute, vol. 355, no. 13, pp. 6045-6061, 2018. 
[22] M. Cao, S. Zhang, and M. K. Camlibel, "A class of uncontrollable diffusively coupled multiagent systems with multichain topologies," IEEE Transactions on Automatic Control, vol. 58, no. 2, pp. 465-469, 2013.

[23] Z. Ji and H. Yu, "A new perspective to graphical characterization of multiagent controllability," IEEE Transactions on Cybernetics, vol. 47, no. 6, pp. 1471-1483, 2017.

[24] H. Su, M. Long, and Z. Zeng, "Controllability of two-timescale discrete-time multiagent systems," IEEE Transactions on Cybernetics, vol. 50, no. 4, pp. 1440-1449, 2020.

[25] B. Liu, H. Su, R. Li, D. Sun, and W. Hu, "Switching controllability of discrete-time multi-agent systems with multiple leaders and time-delays," Applied Mathematics and Computation, vol. 228, pp. 571-588, 2014.

[26] B. Liu, T. Chu, L. Wang, Z. Zuo, G. Chen, and H. Su, "Controllability of switching networks of multi-agent systems," International Journal of Robust and Nonlinear Control, vol. 22, no. 6, pp. 630-644, 2012.

[27] Y. Guan and L. Wang, "Controllability of multi-agent systems with directed and weighted signed networks," Systems \& Control Letters, vol. 116, pp. 47-55, 2018.

[28] J. Yu and L. Wang, "Group consensus in multi-agent systems with switching topologies and communication delays," Systems \& Control Letters, vol. 59, no. 6, pp. 340-348, 2010.

[29] B. Liu, Y. Han, F. Jiang, H. Su, and J. Zou, "Group controllability of discrete-time multi-agent systems," Journal of the Franklin Institute, vol. 353, no. 14, pp. 3524-3559, 2016.

[30] B. Liu, H. Su, F. Jiang, Y. Gao, L. Liu, and J. Qian, "Group controllability of continuous-time multi-agent systems," IET Control Theory \& Applications, vol. 12, no. 11, pp. 1665-1671, 2018. 